\title{
Efficient two-dimensional magnetotellurics modelling using implicitly restarted Lanczos method
}

\author{
Krishna Kumar, Pravin K Gupta and Sri Niwas* \\ Department of Earth Sciences, Indian Institute of Technology Roorkee, Roorkee 247 667, India. \\ *e-mail: srsnpfes@iitr.ernet.in
}

This paper presents an efficient algorithm, FDA2DMT (Free Decay Analysis for 2D Magnetotellurics $(\mathrm{MT})$ ), based on eigenmode approach to solve the relevant partial differential equation, for forward computation of two-dimensional (2D) responses. The main advantage of this approach lies in the fact that only a small subset of eigenvalues and corresponding eigenvectors are required for satisfactory results. This small subset (pre-specified number) of eigenmodes are obtained using shift and invert implementation of Implicitly Restarted Lanczos Method (IRLM). It has been established by experimentation that only 15-20\% smallest eigenvalue and corresponding eigenvectors are sufficient to secure the acceptable accuracy. Once the single frequency response is computed using eigenmode approach, the responses for subsequent frequencies can be obtained in negligible time. Experiment design results for validation of FDA2DMT are presented by considering two synthetic models from COMMEMI report, Brewitt-Taylor and Weaver (1976) model and a field data based model from Garhwal Himalaya.

\section{Introduction}

Magnetotellurics (MT) method is a natural source electromagnetic method first investigated independently by Cagniard (1953) as well as Tikhonov (1950). The method depends on electromagnetic (EM) wave frequency and physical property of the earth and is based on skin-depth phenomenon. In skin-depth phenomenon, the penetration depth is a function of wave frequency. As the frequency decreases, the depth of penetration increases providing better information (in terms of signal to noise ratio) of earth properties at greater depth.

The geophysical inverse problem consists of determining material properties of the earth's interior from data usually obtained on the surface. Likewise, MT data inversion means determination of electrical resistivity variation in the crust and upper mantle from surface data. However, like any inversion, a pre-requisite for MT data inversion is the solution of corresponding forward problem obtainable by solving the relevant partial differential equation. The solution of such problem needs continuous description of the system that implies an infinite number of elements. Due to computational limitations, such continuous problems can only be solved exactly by mathematical techniques. The available analytical techniques usually limit the possibilities to grossly simplified situations. The simplest parameterization is the resistivity variation only with depth (1D). For solving $2 \mathrm{D}$ and $3 \mathrm{D}$ problems one has to opt for approximate solutions obtained using an appropriate numerical technique such as finite difference method, finite element method or integral equation method (Weaver 1994; Gupta et al 1999; Mitsuhata and Uchida 2004; Wannamaker 1991; etc.). Due to easier implementation of finite difference in comparison with the finite element and integral equation methods, we have opted for the

Keywords. Finite difference; eigenmode method; multi-frequency approach. 
finite difference numerical method (FDM) in the present study.

The first FDM-based 2D forward modelling program was developed by Jones and Pascoe (1971). Since then, several researchers have contributed to the refinement of the finite difference solution of $2 \mathrm{D}$ induction problems. For solving the resulting matrix equation, different methods (LU decomposition and Gauss elimination, etc.) have been used for the single-frequency solution. Park and Chave (1984) used Singular Value Decomposition (SVD) for $2 \mathrm{D}$ forward modelling. However, the versatile eigenmode method having proficiency for multifrequency solution has not been applied in the MT forward modelling as yet. This method was introduced in the context of helicopter-borne electromagnetics (Christiane Stuntebeck 2003). Druskin and Knizhnerman (1994) and Druskin et al (1999) had earlier shown that it is possible to obtain the multi-frequency response at almost the same computational cost than for a single frequency using Lanczos method. In eigenmode approach, Finite Difference (FD) formulation is used, where the domain of study is discretized to transform the governing Helmholtz's equation into a matrix equation. Air half space is incorporated through integral boundary condition. This matrix is transformed into a symmetric matrix because the latter has real eigenvalues. The eigen analysis of this real symmetric matrix is performed. The physics of the problem suggests that only a small subset of small eigenvalues and corresponding eigenvectors need to be considered in the analysis. Hence, invert mode of Implicitly Restarted Lanczos Method (IRLM) (Sorensen 1992) has been used to perform such eigen analysis. Bi-Conjugate Gradient Stabilized (BiCGSTAB) method is used in shift and invert mode to get a faster convergence.

\section{Mathematical formulation}

Like any EM phenomenon, MT field is also governed by Maxwell's equations, which can be transformed for the frequency range used in MT and for the conductivity range of the medium, into the following vector diffusion equation

$$
\nabla \times \nabla \times \mathbf{E}+\mu_{0} \sigma \frac{\partial \mathbf{E}}{\partial t}=0
$$

Here, $\mathbf{E}$ is the electric field intensity vector, $\mu_{0}$ is the magnetic permeability in free space and $\sigma$ is the conductivity function in the study domain.

Assuming harmonic time dependence, $\exp (i \omega t)$, equation (1) reduces to the vector Helmholtz equation

$$
\nabla \times \nabla \times \mathbf{E}+i \omega \mu_{0} \sigma \mathbf{E}=\mathbf{J}
$$

where $\omega$ is the angular frequency of the harmonic field and $\mathbf{J}$ is the impressed currents defined in terms of the imposed boundary conditions.

In conventional MT, modelling equation (2) is numerically solved for different frequencies independently. This means that the computation of multi-frequency (say $m$ frequencies) response generation will be $m$ times that of a single frequency response. In order to employ Free Decay Mode Analysis, let us assume the time dependence $\exp (-\lambda t)$, then equation (1) reduces to the following equation describing the eigen problem,

$$
\nabla \times \nabla \times \mathbf{e}(\mathbf{r})=\lambda \mu_{0} \sigma \mathbf{e}(\mathbf{r}) .
$$

Here, $\lambda$ is the decay constant and $\mathbf{e}(\mathbf{r})$ is the spatial dependence of the field eigenvector.

Equation (3) is a generalized eigen problem that can be solved numerically. Using FDM, the problem described by equation (3) can be visualized as a matrix eigenvalue problem,

$$
A e_{n}=\lambda_{n} e_{n}, \quad n=1,2, \ldots, N,
$$

where $A$ is the $N \times N$ coefficient matrix whose elements depend on geometry and conductivity distribution, $\lambda_{n}$ are eigenvalues and $e_{n}$ are respective eigenvectors. Here, $N$ is the number of internal nodes in the grid or the number of unknowns.

Electric field solution to equation (2), represented as a superposition of free decay mode eigenvectors, can be expressed as:

$$
\mathbf{E}(\mathbf{r}, \boldsymbol{\omega})=\sum_{n} a_{n}(\omega) \mathbf{e}_{n}(\mathbf{r}) .
$$

The coefficients $\left(a_{n}\right)$ are obtained by exploiting $\sigma$ orthogonality of the eigenvectors

$$
\int_{V} \sigma \mathbf{e}_{m}(\mathbf{r}) \cdot \mathbf{e}_{n}(\mathbf{r}) d^{3} r=\delta_{m n},
$$

and using equations (2), (3) and (5). The resulting expression is given by

$$
a_{n}(\omega)=\frac{1}{\mu_{0}\left(\lambda_{n}+i \omega\right)} \int_{v} \mathbf{J}(\mathbf{r}) \cdot \mathbf{e}_{n}(\mathbf{r}) d^{3} r .
$$

These $\left(a_{n}\right)$ are used to solve for the electric field values using equation (5). Magnetic field is obtained by Maxwell's equation of Faraday's law. MT response functions, such as impedance, apparent resistivity and phase, are obtained by using the electric and magnetic fields in appropriate relations.

Since $\lambda_{n}$ is in the denominator in equation (7), main contribution to electric field comes from small eigenvalues $(\lambda)$ and corresponding eigenvectors. So, only a subset of small eigenvalues is sufficient for an acceptable solution. One of the methods to find 
this subset is the Lanczos method which is briefly discussed below.

\subsection{Lanczos method}

Lanczos method is based on the Krylov subspace technique. Krylov subspace can be obtained by any arbitrary vector $\left(\mathbf{V}_{1}\right)$ and its repeated product with the system matrix $\mathbf{A}$. The theory of Lanczos method and the programs for a symmetric matrix are described in Cullum and Willoughby (1985a, 1985b). The Krylov basis vectors are not orthogonal. The orthogonalized basis vectors obtained from the Krylov vectors serve as Lanczos vectors. Initially, Lanczos method was used to solve for all eigenvalues and eigenvectors of a symmetric matrix using

$$
\mathbf{A V}=\mathbf{V H}+\mathbf{r}
$$

where $\mathbf{A}$ is the original matrix, $\mathbf{V}$ is the matrix of Lanczos vectors of dimension $N$ columnwise, $\mathbf{H}$ is a symmetric tri-diagonal matrix and $\mathbf{r}$ is a residual vector. All matrices are $N \times N$ order and vector $\mathbf{r}$ is an $N$ dimensional vector.

Complete eigen analysis of the symmetric tridiagonal matrix $\mathbf{H}$ needs to be performed. This, in turn, leads to eigenvectors of matrix A. Theoretically, $\mathbf{r}$ should be zero but in finite precision arithmetic it has a non-zero value albeit very small.

Presently, Lanczos method is widely used to find a desired subset of eigenvalues as described by Sorensen (1992). In our problem, we are interested in $M$ number of smallest eigenvalues and corresponding eigenvectors. After $M$ number of Lanczos vectors generation, the residual vector has a finite value. To find $M$ number of smallest eigenvalues and corresponding eigenvectors, we have to generate a subspace with $(M+P)$ number of Lanczos vectors as given below

$$
\mathbf{A} \mathbf{V}_{M+P}=\mathbf{V}_{M+P} \mathbf{H}_{M+P}+\mathbf{r}_{M+P} e_{M+P}^{T}
$$

In this case, the $\mathbf{V}$ matrix is of the order of $N \times(M+P)$ and $\mathbf{H}$ matrix is of the order of $(M+P) \times(M+P)$. Eigen analysis of this smaller dimensional matrix $\mathbf{H}$ is performed rather than full $N \times N$ matrix. The eigenvalues obtained from this $M+P$ factorization reflects characteristics of full spectrum of $N$ eigenvalues. We arrange the $M+P$ eigenvalues in increasing order and the last $P$ largest eigenvalues are the unwanted ones. These $P$ eigenvalues are used as shifts to update the first $M$ eigenvalues via $\mathrm{QR}$ method (successive Householder transformations) (Golub and Van Loan 1983). In this updating process Lanczos vectors are forced into the subspace corresponding to smallest eigenvalues and the residual vector $\mathbf{r}_{M+P}$ becomes small iteratively. This technique is known as Implicit Restart Technique. It is recommended that $P$ be greater than $M$.

With this restarting technique we can compute the largest eigenvalues more efficiently. Therefore, the invert mode is used to find the smallest eigenvalues. In invert mode, Krylov vectors are generated with $A^{-1}$ instead of $A$. To solve $A^{-1}$ we need an efficient solver and one such method is BiCGSTAB method.

\section{Development of FDA2DMT algorithm}

The 2D algorithm FDA2DMT, based on eigenmode analysis using FDM, is developed. Initially Dirichlet boundary conditions are applied on the domain boundaries - two horizontal and two vertical sides. The problem is solved for internal nodes only using a manual grid and total field formulation. All the eigenmodes are computed and used to synthesize the electric field. The results are satisfactory at the centre of the model, however, unsatisfactory near the vertical domain boundaries. This problem is circumvented by using a skin-depth field decay criterion based autogrid along with a mixture of Dirichlet's and Neumann's boundary conditions.

Further, the application of integral boundary condition at the air-earth interface (Stuntebeck 2003) and of asymptotic boundary condition (Weaver and Brewitt-Taylor 1978) at the vertical sides yielded accurate results at the vertical boundaries. To start with, the responses are computed using all eigenmodes followed by the Implicitly Restarted Lanczos method (IRLM) (Sorensen 1995; Lehoucq et al 1997) for the computation of the responses at different percentage of eigenmodes. IRLM works accurately in regular mode (largest eigenmodes) and in invert mode (smallest eigenmodes). Our interest lies in the computation of smallest eigenmodes for which IRLM is used in invert mode. In invert mode, a matrix solver is needed as the Lanczos basis vectors are computed using $A^{-1} v$. Different methods based on conjugate gradient with and without preconditioner are tested and the efficiency is shown in table 1. BICGStab is preferred over other methods as an inverse matrix solver with preconditioner ILU(0) to compute the Lanczos basis vectors in invert mode. Since in MT formulation, the derived observables: impedance, apparent resistivity and phase, depend on the ratio of orthogonal horizontal components of $\mathbf{E}$ and $\mathbf{H}$ field values, we verified that these responses are identical in two cases where field boundary conditions are expressed either in terms of $\mathbf{E}$ field or $\mathbf{H}$ field. All computations are performed on a Pentium 4.0 Processor with 1 GB RAM. 
Table 1. Comparison of different methods with preconditioners for best invert matrix solver.

\begin{tabular}{lc}
\hline Method & $\begin{array}{c}\text { Number of } \\
\text { iterations }\end{array}$ \\
\hline CG & 30 \\
BiCG & 20 \\
BiCGStab & 15 \\
CG + ILU $(0)$ & 12 \\
BiCGStab + ILU $(0)$ & 9 \\
\hline
\end{tabular}

\section{Algorithm testing}

It is desirable that an algorithm is exhaustively validated for analytical solution. In this context, we first tested the developed algorithm for uniform half space. The numerical results are identical almost everywhere with the analytical results. Multi-frequency responses, generated with eigenmodes of single frequency, match with the corresponding analytical results.

\subsection{Experimental results}

The next validation of this algorithm is the reproduction of established published results. In this exercise, we have selected two 2D models from COMMEMI (Comparison of Modeling Methods for Electro-Magnetic Induction) report (Zhdanov et al 1997), one simple model (2D-1) and the other a complex (2D-4) one. In COMMEMI report the results obtained using different algorithms based on Finite Difference, Finite Element, and Integral Equation methods for same models are given. We are presenting the comparison for electric field and apparent resistivity values only.

In COMMEMI report (Zhdanov et al 1997), the electric field, magnetic field and apparent resistivity values are given, not the phase values. We are comparing the electric field and apparent resistivity values because we think if electric field and apparent resistivity are accurate then magnetic field is automatically accurate. As we know, MT apparent resistivity is the ratio of electric and magnetic fields. Phase values are not presented here because in the COMMEMI report phase values are not described. For uniformity, we present same MT response plots for all models as for test models of COMMEMI.

\subsubsection{Simple model}

Model 2D-1 in the COMMEMI report is reproduced in figure 1(a). It comprises a symmetrical rectangular insert embedded in homogeneous half space. The resistivity of the inserted block is $0.5 \Omega \mathrm{m}$ while that of the half space is $100 \Omega \mathrm{m}$. The block, placed at a depth of $250 \mathrm{~m}$ from the earth surface, has a width of $1 \mathrm{~km}$ and thickness of $2 \mathrm{~km}$. The MT responses such as electric field and apparent resistivity are computed for a period of $0.1 \mathrm{~s}$. The number of nodes in $\mathrm{y}$ and $\mathrm{z}$ directions is 51 and 50, respectively; the total number of unknowns is 2550. FDA2DMT took 1:32 hr to compute the response using all eigenmodes. In figure 1(b), the eigenvalue plot for the model is presented while

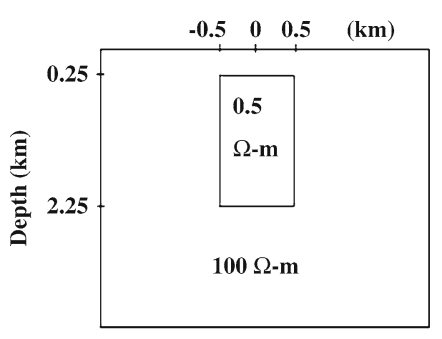

(a)

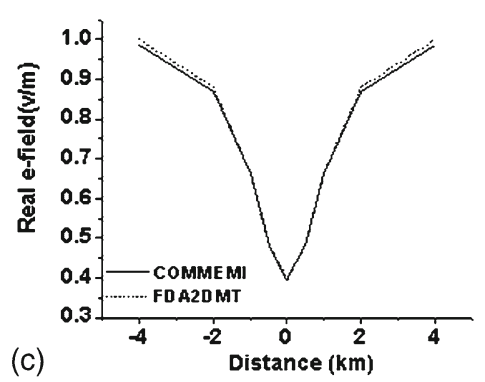

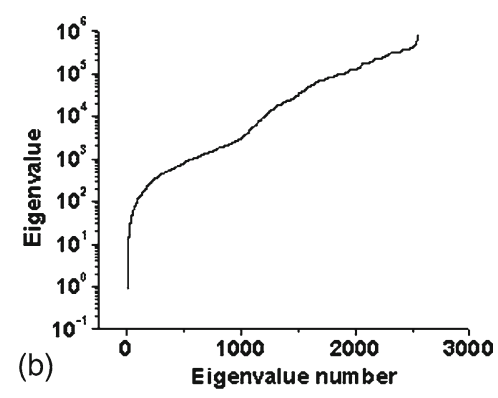

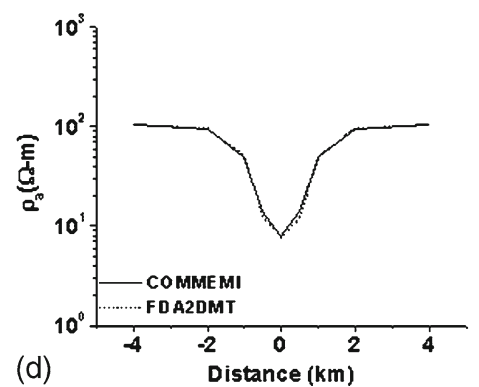

Figure 1. (a) Simple model (Model 2D-1 of COMMEMI, distances in $\mathrm{km}$ and resistivity in $\Omega \mathrm{m}$ ), (b) eigenvalue plot; comparison of COMMEMI (Zhdanov et al 1997) and FDA2DMT at $0.1 \mathrm{~s}$, (c) electric field, and (d) apparent resistivities. 
in figure 1(c and d) electric field and apparent resistivity responses, respectively. They are compared with the average values given in the COMMEMI report. The RMS error between the two responses is $1 \%$.

\subsubsection{Complex model}

Model 2D-4 is reproduced in figure 2(a). It consists of different blocks with resistivity ranges between 2.5 and $1000 \Omega \mathrm{m}$ having different width and thicknesses. Zhdanov et al (1997) observed that for this model the minimum misfit is at $1 \mathrm{~s}$. They also stated that the misfit in apparent resistivities from different algorithms varies in the range of $5-10 \%$. The number of nodes in $\mathrm{y}$ and $\mathrm{z}$ directions is 66 and 54 respectively and the total number of unknowns is 3564 . FDA2DMT took $2: 30 \mathrm{hr}$ to compute the

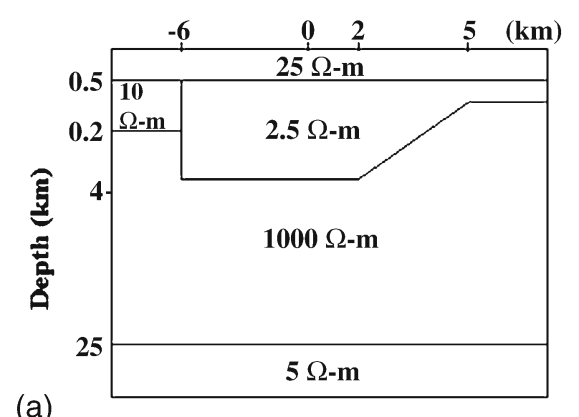

(a)
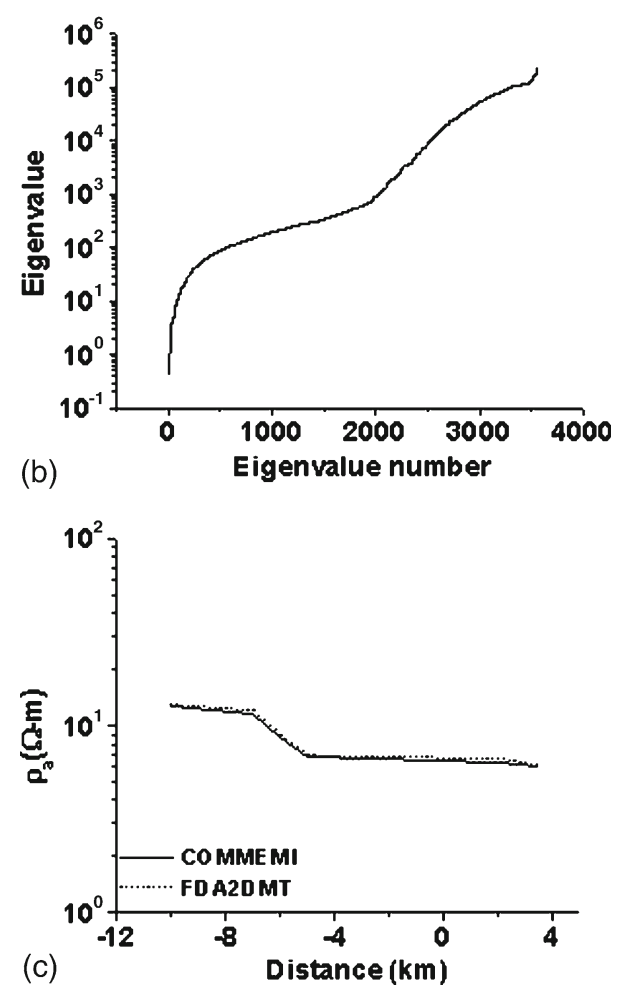

Figure 2. (a) Complex model (Model 2D-4 of COMMEMI, distances in $\mathrm{km}$ and resistivity in $\Omega \mathrm{m}$ ), (b) eigenvalue plot, and (c) comparison between apparent resistivities of COMMEMI and FDA2DMT at $1 \mathrm{~s}$. response using all eigenmodes. The eigenvalue plot for the complex model is given in figure 2(b). The apparent resistivity response of this model is compared in figure 2(c) with the average values published in the COMMEMI report. The relative RMS error in apparent resistivity is $6 \%$, which is within the acceptable limits. We have observed that our results are closer to the Integral Equation method results.

After achieving good response using all eigenmodes, we checked whether a subset of smallest eigenmodes is sufficient to obtain accurate responses. This important and far reaching exercise is described in the following.

\subsubsection{Effect of different percentage of eigenmode}

In order to perform the experiment with subsets of eigenmodes in terms of percentages, BrewittTaylor and Weaver (1976) model has been used. It consists of a $0.1 \Omega \mathrm{m}$ cube inserted in a $1 \Omega \mathrm{m}$ half space at $100 \mathrm{~m}$ depth. The model is shown in figure $3(\mathrm{a})$. The number of nodes in $\mathrm{y}$ and $\mathrm{z}$ directions is 34 and 30 respectively; total number of unknowns is 1020. FDA2DMT took 4:58 min to compute for response using all eigenmodes. Figure 3(b) represents the eigenvalue plot. The number of eigenmodes used to compute MT responses in 5, 10, 15 and $20 \%$ are 51, 120, 153 and 204 and the time to compute the responses is $9 \mathrm{~s}, 23 \mathrm{~s}, 49 \mathrm{~s}$ and 1:52 min, respectively. For different percentages of computed eigenmodes, the electric fields and apparent resistivities are given in figure $3(\mathrm{c}$ and $\mathrm{d}$ ) respectively. The response curves for the four eigenmode percentages $(5,10,15$ and 20$)$ match well with the results computed using all the eigenmodes. It is heartening to note that even $5 \%$ of eigenmodes are sufficient for accurate field synthesis. This study was only on the conductive block. Next study is performed to observe the effect of conductive body along with the resistive body.

For this purpose, the model described in COMMEMI report as 2D-3, is considered. In this model, the resistive and conductive blocks are exposed at the surface. The model is shown in figure 4(a). The number of nodes in $\mathrm{y}$ and $\mathrm{z}$ directions are 60 and 30 respectively; total number of unknowns are 1800. FDA2DMT took 21:29 min to compute for response using all eigenmodes. The eigenvalue plot for the model is presented in figure 4(b). To compute 5, 10, and 20\% eigenmodes, the number of eigenmodes used are 90, 180 and 360 and the time to compute the responses is $40 \mathrm{~s}, 1: 45 \mathrm{~min}$ and 3:14 min respectively. The response curves for $10 \mathrm{~s}$ period are presented for 5, 10 and $20 \%$ of eigenmodes. Electric field and apparent resistivity curves for different percentages are presented in figure 4(c and d) respectively. In conductive block 


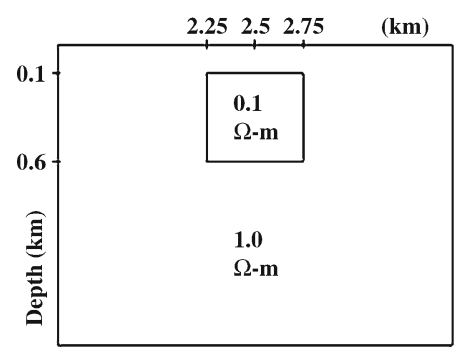

(a)

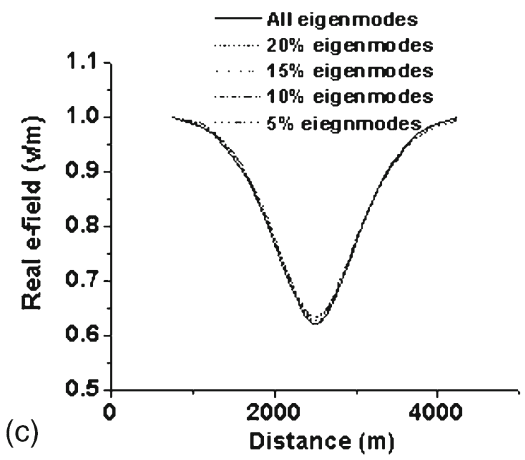

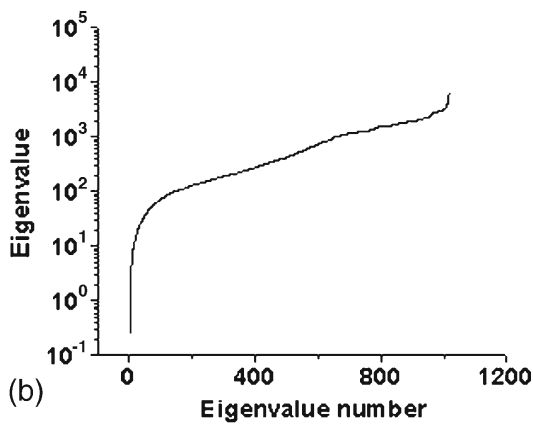

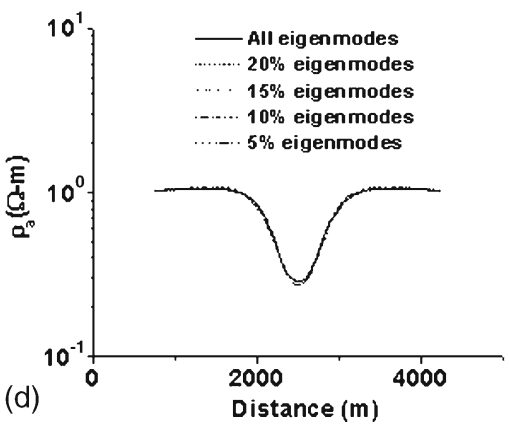

Figure 3. (a) 2D Test model (distances in $\mathrm{km}$ ), (b) eigenvalue plot, plots for different percentage of eigenmodes, (c) reelectric field, and (d) apparent resistivities.

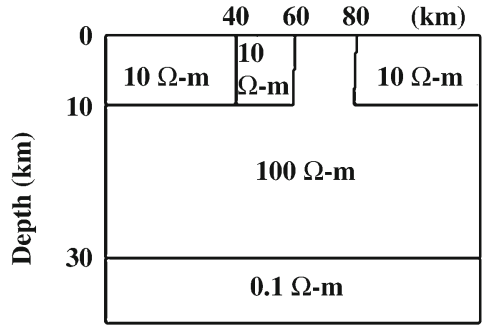

(a)

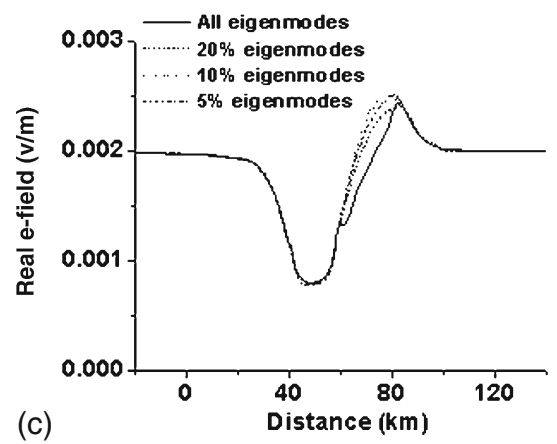

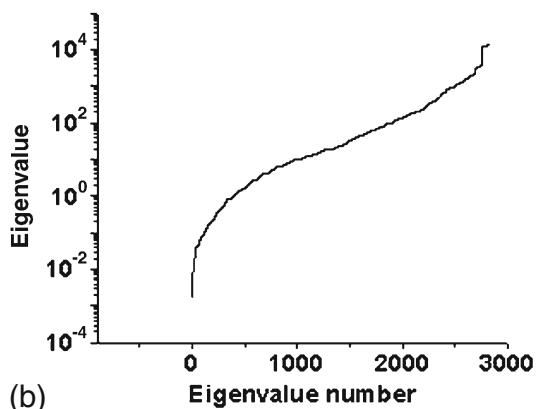

(b)

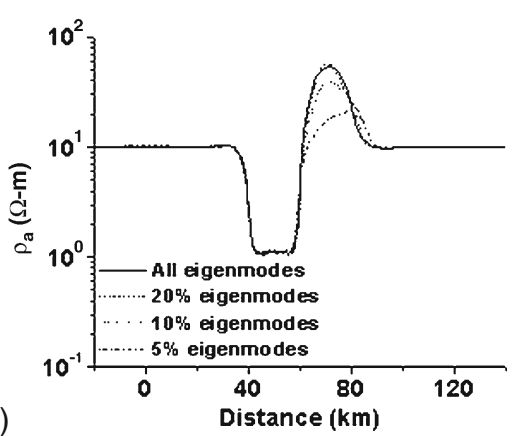

Figure 4. (a) 2D-3 model of COMMEMI, (b) eigenvalue plot, plots for different percentage of eigenmodes at $10 \mathrm{~s}$, (c) real e-field, and (d) apparent resistivity.

even $5 \%$ eigenmodes are sufficient but in resistive block case, $20 \%$ eigenmodes gives satisfactory results. Thus, for conductive block smaller numbers of eigenmodes are sufficient while for resistive block a larger number of eigenmodes are required. The relative RMS errors for 5, 10 and 20\% eigenmode results compared to all eigenmodes response are $25.5,12.2$ and $5.5 \%$ respectively.

We have selected the $5 \%$ and $20 \%$ criteria because in less than $5 \%$ the MT response such as electric field is not accurate enough and above $20 \%$, the MT response such as electric field is better but 

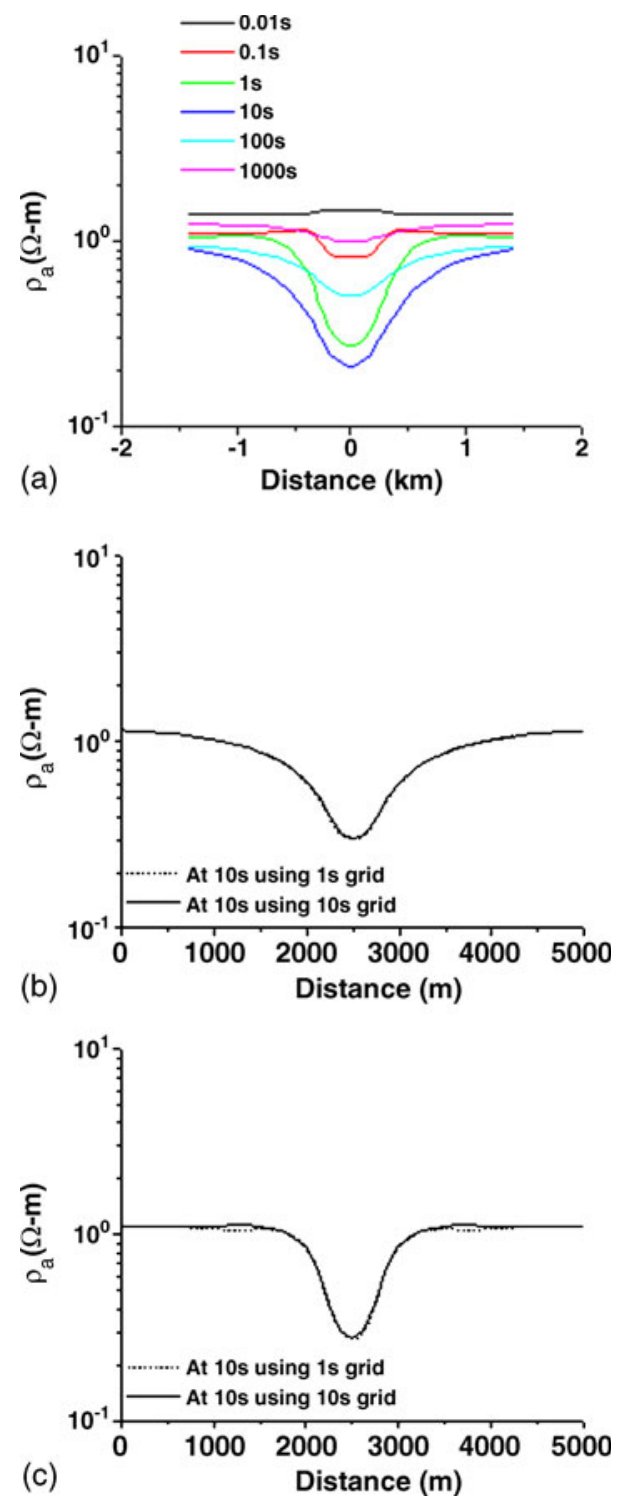

Figure 5. Response curves for multi-frequencies (a) from $0.01 \mathrm{~s}$ to $1000 \mathrm{~s}$ using grid at $1 \mathrm{~s},(\mathbf{b})$ at $1 \mathrm{~s}$, and (c) at $10 \mathrm{~s}$.

the algorithm took more time to get the response. Thus $5 \%$ and $20 \%$ is the optimized value to get accurately enough response in less time.

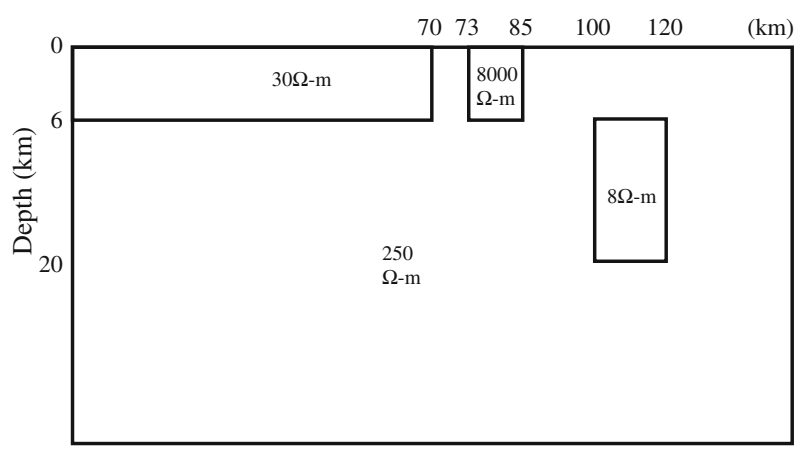

Figure 7. Simplified geoelectrical model of Tyagi (2007).

\subsubsection{Multi-frequency experiment}

The multi-frequency experiment is performed on the Weaver model (1978) with the grid at $1 \mathrm{~s}$. The responses, computed at $0.01 \mathrm{~s}, 0.1 \mathrm{~s}, 1 \mathrm{~s}, 10 \mathrm{~s}, 100 \mathrm{~s}$ and $1000 \mathrm{~s}$ by reusing the only $5 \%$ computed eigenmodes, are shown in figure 5(a). In computing only $5 \%$ eigenmodes the algorithm takes only $17 \mathrm{~s}$ for $34 \times 32$ grid with total 1088 unknowns while for all eigenmodes it takes 7:52 min. For each frequency computations the algorithm takes only a fraction of second. The results are presented for 6 time periods and from figure 5(a) it is clear that as time period increases from $0.01 \mathrm{~s}$ to $1 \mathrm{~s}$, the response become strong from flat curve and again as time period increases the response become flat as we have crossed the body. Thus in sounding experiments the algorithm proves it efficacy.

In order to study the accuracy of multi-frequency response computations using eigenmodes, the grids are generated corresponding to two time periods $1 \mathrm{~s}$ and $10 \mathrm{~s}$ and computed the responses for the two time periods using each one of these grids. The comparison of the response values for $1 \mathrm{~s}$ and $10 \mathrm{~s}$ time periods obtained using both the grids are respectively shown in figure 5 (b and $\mathrm{c}$ ). The relative RMS error with respect to true response for $1 \mathrm{~s}$ response is $1.4 \%$ and for $10 \mathrm{~s}$ is $0.8 \%$. Hence, the multi-frequency responses computed using eigenmodes are quite accurate.

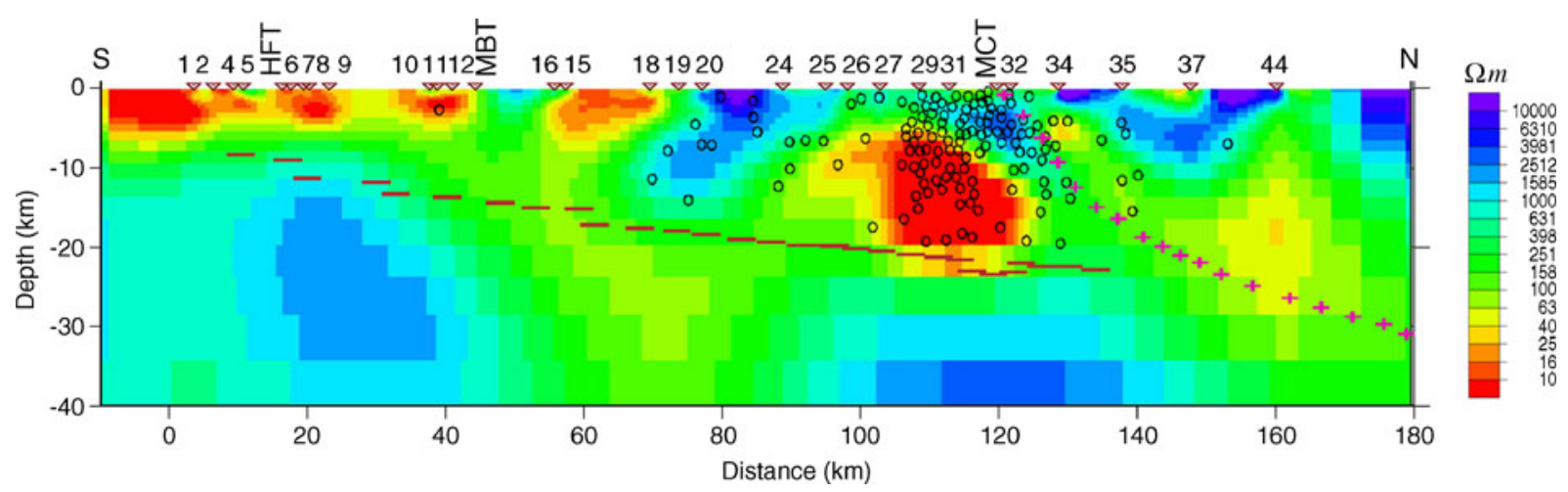

Figure 6. 2D resistivity models of the crust derived from inversion of joint TE-TM mode MT data (Tyagi 2007). 

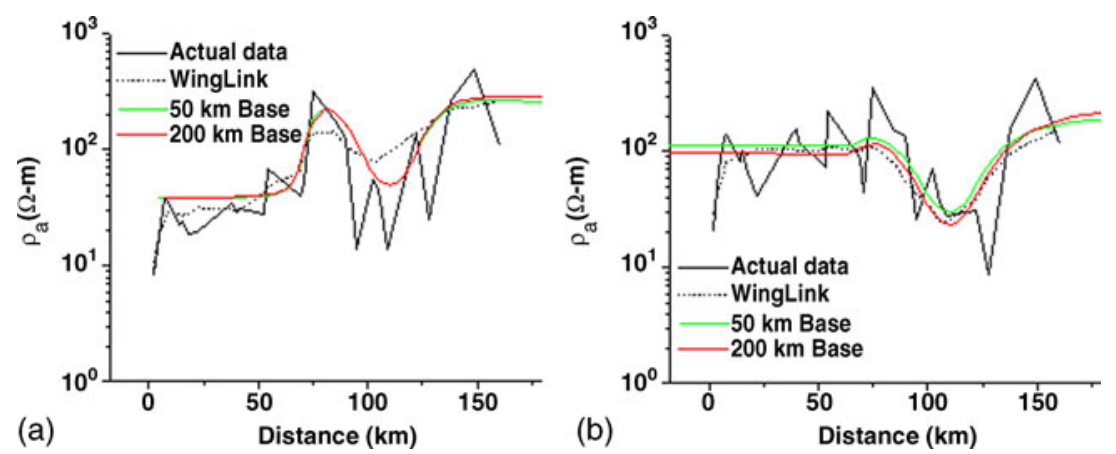

Figure 8. 2D plot with different basement (a) at $11.61 \mathrm{~s}$ and (b) at $90.45 \mathrm{~s}$.
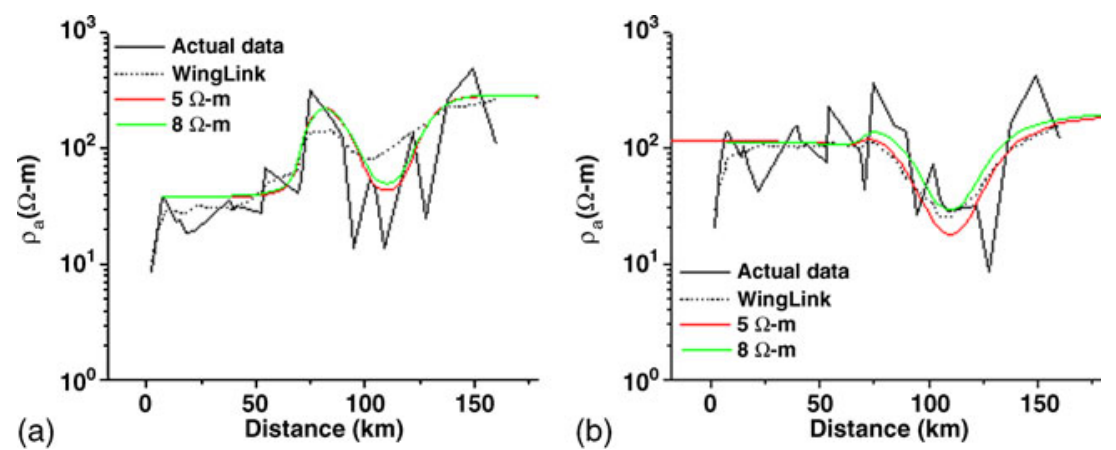

Figure 9. 2D plot with resistivity variation of conductive block (a) at $11.61 \mathrm{~s}$ and (b) $90.45 \mathrm{~s}$.

\subsection{Experiment with field data}

A geoelectrical model based on field data from Garhwal Himalaya (Roorkee to Gangotri profile) is considered for this study. The simplified model from the complex 2D model (figure 6) proposed by Tyagi (2007) and Israil et al (2008) based on observed MT data in the area is shown in figure 7 . To match the computed $2 \mathrm{D}$ responses with the field data and with the inverted model response, the model parameters are tuned. Multi-frequency responses were compared with corresponding field data. From the time period list of MAPROS processing software, we chose two time periods $11.61 \mathrm{~s}$ and $90.45 \mathrm{~s}$ so that we can compare our response values with actual field data. Different experiments designed for the $2 \mathrm{D}$ case are:

- study with different basement depths,

- study with different block resistivities,

- multi-frequency responses,

- comparison with and without salient features.

\subsubsection{Study with different basement depths}

We have assumed perfectly conducting basement $(0.001 \Omega \mathrm{m})$ and the host resistivity is $250 \Omega \mathrm{m}$. In order to study the effect of this conductive basement, we experimented with two basement depth values of $50 \mathrm{~km}$ and $200 \mathrm{~km}$. The number of nodes for $11.61 \mathrm{~s}$ at $50 \mathrm{~km}$ and $200 \mathrm{~km}$ basement depth are $(49,25)$ and $(49,35)$ in $(y, z)$ directions respectively and the total number of unknowns are 1225 and 1715. FDA2DMT took 9:35 min and 21:17 min to compute for response using all eigenmodes. The number of nodes for $90.45 \mathrm{~s}$ at $50 \mathrm{~km}$ and $200 \mathrm{~km}$ basement depth are $(41,15)$ and $(41,22)$ in $(y$, $\mathrm{z}$ ) directions respectively and the total number of unknowns are 615 and 902. Algorithm took 1:37 min and 4:19 min to compute for response using all eigenmodes. We observe that at $11.61 \mathrm{~s}$ the basement depth has no effect, as the $50 \mathrm{~km}$ and $200 \mathrm{~km}$ curves overlap (figure 8a). However, at $90.45 \mathrm{~s}$ period the effect of basement depth is clearly visible where the two curves are separated apart (figure 8b). Thus the basement depth is finalized as $200 \mathrm{~km}$.

\subsubsection{Study with different block resistivities}

Subsequently we varied the resistivity of conductive block to fix the appropriate resistivity of blocks. The responses are compared in figure 9 (a and b) at $11.61 \mathrm{~s}$ and $90.45 \mathrm{~s}$ periods, respectively. Our main emphasis is on the study of the conductive feature. We carried out the experiment with resistivity value of this block as 5 and $8 \Omega \mathrm{m}$ keeping other parameters unchanged. The relative RMS error is $21 \%$ and $19 \%$ at $11.61 \mathrm{~s}$ and $11 \%$ and $9 \%$ 

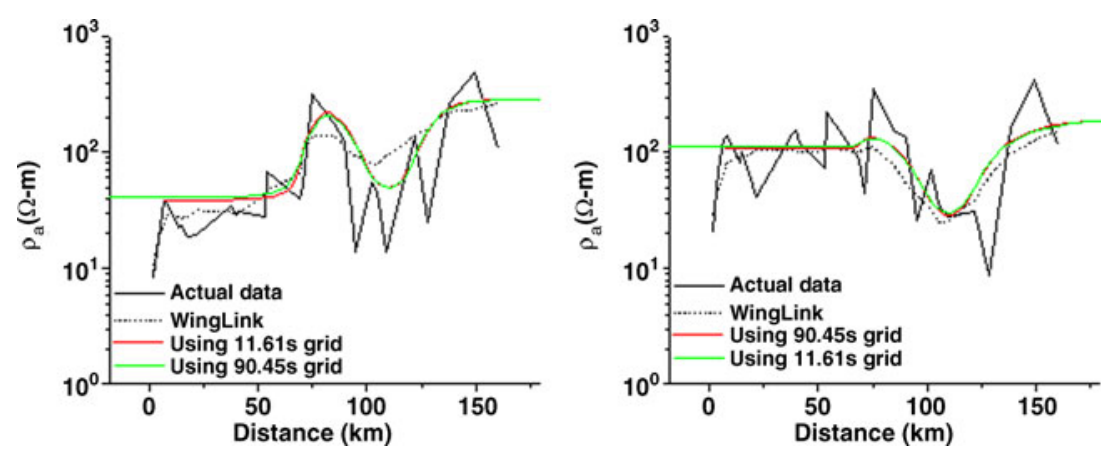

Figure 10. Response curves for multi-frequency using eigenmodes of $11.61 \mathrm{~s}$ and $90.45 \mathrm{~s}$ grid (a) at $11.61 \mathrm{~s}$ and (b) at $90.45 \mathrm{~s}$.

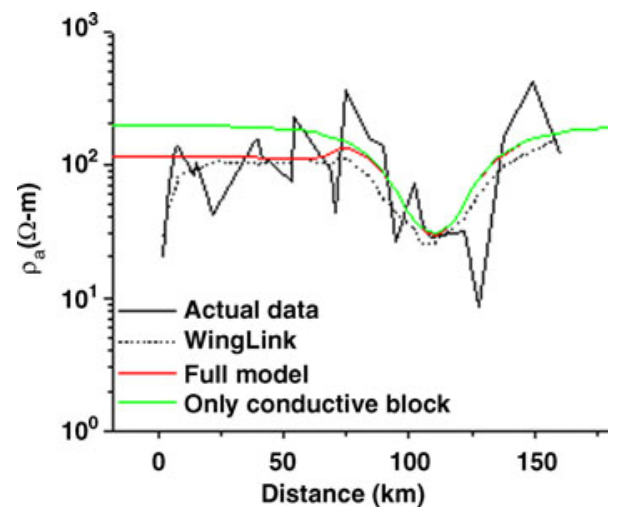

Figure 11. Curves with and without the features other than conductor.

at $90.45 \mathrm{~s}$ for 5 and $8 \Omega \mathrm{m}$ respectively. Therefore, $8 \Omega \mathrm{m}$ resistivity of the conductivity block is used for further modelling.

\subsubsection{Multi-frequency responses}

In this experiment two grids of the complex Gangotri model are used and the responses are computed at one period using other period grid to confirm that the responses are sufficiently accurate at other period grid in complex domain. Two grids for periods $11.61 \mathrm{~s}$ and $90.45 \mathrm{~s}$ were generated for this study. The responses at $11.61 \mathrm{~s}$ using the $11.61 \mathrm{~s}$ grid and the $90.45 \mathrm{~s}$ grid are compared with each other and with the field data and WinGLink response (the response of the inverted model of field data with the software provided by Metronix) in figure $10(\mathrm{a})$. The relative RMS error is $0.9 \%$ at $11.61 \mathrm{~s}$ and $0.3 \%$ at $90.45 \mathrm{~s}$ using $11.61 \mathrm{~s}$ grid and $90.45 \mathrm{~s}$ grid respectively. In figure $10(\mathrm{~b})$, the $90.45 \mathrm{~s}$ response obtained using the $11.61 \mathrm{~s}$ grid and the $90.45 \mathrm{~s}$ grid are compared and found to match well with each other and also with the corresponding field data. This experiment inspires confidence that the multi-frequency solution is not only accurate for simple geometry models but also for complex models.

\subsubsection{Comparison with and without salient features}

Since we are interested in the conductive feature and we intend to use $90.45 \mathrm{~s}$ period in $3 \mathrm{D}$ modelling, the effect of other features (such as conductive layer and resistive block) on the response must be tested at this period only. We removed these features one by one and compared the responses with the response of model having only the conductive block. The response curves plotted in figure 11 reveal that the curve segment over the conductive block is not affected by other features. Thus, the $2 \mathrm{D}$ experiments reveal that study of single block having resistivity $8 \Omega \mathrm{m}$ and basement located at $200 \mathrm{~km}$, for time period $90.45 \mathrm{~s}$ is good enough for designing 3D models. The 3D nature of this conductive block can be studied as if other features are not there.

\section{Conclusion}

The 2D MT forward modelling algorithm FDA2DMT which employs Finite Difference Method (FDM) and an eigenmode approach (EA) is developed and validated. The validation exercise reveals that only $15-20 \%$ smallest eigenvalues are sufficient for accurate field synthesis and these are efficiently determined using Implicitly Restarted Lanczos Method (IRLM). The test results for models chosen from COMMEMI report (Zhdanov et al 1997) and from Brewitt-Taylor and Weaver (1976) are presented, and they match well with the published values. On the basis of the theoretical strength of eigenmode approach and the success of numerical experiments, it can be concluded that the eigenmode approach is efficient for modelling of MT data. This algorithm is particularly useful for computation of multi-frequency response. The multifrequency computations are verified on BrewittTaylor and Weaver (1976) model and the responses of two grids are also compared on the other period grid on the complex model based on field data to verify the accuracy of the results. This approach 
is good if someone is interested in multi-frequency responses as needed in sounding, but not optimal if someone has interest only in single frequency solution. For 3D study of the model proposed by Tyagi (2007), the mid crustal conductor is a prominent feature and it can be studied as a single block without affecting the response of other features.

\section{Acknowledgements}

The authors would like to thank late Prof. Peter Weidelt, Technical University of Braunschweig, Germany, for his suggestions during discussions. Financial support given (to KK) by Ministry of Human Resources and Development (MHRD), India and by KMS Technologies, Houston, USA, are highly acknowledged. The authors are also thankful to the anonymous reviewers for their critical review.

\section{References}

Brewitt-Taylor C R and Weaver J T 1976 On the finite difference solution of two-dimensional induction problems; Geophys. J. R. Atsr. Soc. 47 375-396.

Cagniard L 1953 Basic theory of the magnetotelluric method of geophysical prospecting; Geophys. 18 605-635.

Cullum J K and Willoughby R A 1985a Lanczos algorithms for large symmetric eigenvalue computations; Vol. I, Theory; (Germany: Birkhauser), pp. 268.

Cullum J K and Willoughby R A 1985b Lanczos algorithms for large symmetric eigenvalue computations; Vol. II, Programs (Germany: Birkhauser), pp. 496.

Druskin V and Knizhnerman L 1994 Spectral approach to solving three-dimensional Maxwell's equations in the time and frequency domains; Radio Science 29 937-953.

Druskin V L, Knizhnerman L A and Lee P 1999 New spectral Lanczos decomposition method for induction modeling in arbitrary 3-D geometry; Geophys. 64 701-706.

Gupta P K, Niwas S and Rastogi A 1999 EM2INV - A finite difference based algorithm for two-dimensional inversion of geoelectromagnetic data; Earth Planet. Sci. 108 $233-253$.

Golub G H and Van Loan C F 1983 Matrix Computations; North Oxford Academic, Oxford, pp. 476.
Israil M, Tyagi D K, Gupta P K and Niwas S 2008 Magnetotelluric investigations for imaging electrical structure of Garhwal Himalayan corridor, Uttarakhand, India; J. Earth. Syst. Sci. 117 189-200.

Jones F W and Pascoe L J 1971 A general computer program to determine the perturbation of alternating electric currents in a two-dimensional model of a region of uniform conductivity with an embedded inhomogeneity; Geophys. J. R. Astr. Soc. 24 3-30.

Lehoucq R B, Sorensen D C and Yang C 1997 ARPACK Users' Guide: Solution of Large Scale Eigenvalue Problems with Implicitly Restarted Arnoldi Methods; http:// www.caam.rice.edu/software/ARPACK/UG/ug.html.

Mitsuhata Y and Uchida T 2004 Three-dimensional magnetotelluric modeling using the $\mathrm{T}-\Omega$ finite element method; Geophys. 69 108-119.

Park J and Chave A D 1984 On the estimation of magnetotelluric response functions using the singular value decomposition; Geophys. J. R. Atsr. Soc. 77 683-709.

Sorensen D C 1992 Implicit application of polynomial filters in a k-step Arnoldi method; SIAM J. Matrix Anal. Appl. 13 357-385.

Sorensen D C 1995 Implicitly restarted Arnoldi/Lanczos method for large scale eigenvalue calculations. Parallel Numerical Algorithms; Proceedings of an ICASE/LaRC Workshop (eds) Keyes D E, Sameh A and Venkatakrishnan V, Kluwer.

Stuntebeck C 2003 Three-dimensional electromagnetic modeling by free-decay mode superposition; Ph.D. Thesis, Technical University of Braunschweig, Germany.

Tikhonov A N 1950 On investigation of electrical characteristics of deep strata of Earth's crust (in Russian); Dokl. Acad. Nauk USSR 73 295-297.

Tyagi D K 2007 2D modeling and inversion of magnetotelluric data acquired in Garhwal Himalaya; Ph.D. Thesis, Indian Institute of Technology Roorkee, India.

Wannamaker P E 1991 Advances in three-dimensional magnetotelluric modeling using integral equations; Geophys. 56 1716-1728.

Weaver J T 1994 Mathematical methods for geo-electro magnetic induction; Research Studies Press Ltd., Taunton, Somerset, England.

Weaver J T and Brewitt-Taylor C R 1978 Improved boundary condition for the numerical solution of E-polarization problems in geomagnetic induction; Geophys. J. R. Astr. Soc. 54 309-317.

Zhdanov M S, Varentsov I M, Weaver J T, Golubev N G and Krylov V A 1997 Methods for modeling electromagnetic fields results from COMMEMI-the international project on the comparison of modeling methods for electromagnetic induction; J. Appl. Geophys. 37 133-271. 